\title{
Erratum to: Computational Advances for the Development of Allosteric Modulators and Bitopic Ligands in G Protein-Coupled Receptors
}

\author{
Zhiwei Feng, ${ }^{1,2,3}$ Guanxing Hu, ${ }^{1,2,3}$ Shifan $\mathrm{Ma}^{1,2,3}$ and Xiang-Qun $\mathrm{Xie}^{1,2,3,4,5}$
}

\section{Erratum to: AAPS J}

DOI 10.1208/s12248-015-9776-y

Under the section BITOPIC LIGANDS, the authors have inadvertently omitted the citation of the original work and specific sentences by Mohr et al. (101), who have made significant contributions in this field. The authors regret this error.

Specifically, the following sentence strings should have been placed within quotes and directly attributed to Mohr et al. (101) from which the sentences originated:

“...Steinfeld et al. (94) generated the compound THR160209 by connecting an orthosteric 3-benzyhydrylpyrrolinyl building partner via a heptane chain to an allosteric 4aminobenzylpiperidine motif, and they gained a considerably higher receptor affinity than seen with individual components (pKi (total) $=9.5$ vs. $\mathrm{pKi}=5.5$ for the single compounds) and a certain preference for the M2mAChR" (101).

"This can be achieved if two parts bind into their corresponding binding pockets in an ideal manner without inducing an unfavorable conformational rearrangement of the receptor protein, which can be explained by the lower total entropy cost of the ligand-receptor complex. The mostly favorable spatial proximity between the orthosteric and the allosteric binding areas contributes to a decrease in entropy (96). Conformational changes in the two binding events can either be (locally) entropic or enthalpic. This can differ case by case. The general rule is that an increase in entropy is the driving force for the docking of a polar agonist and a decrease in enthalpy in the driving force for the docking of a mostly hydrophobic antagonist (97)"(101).

"...several parts of the molecule-the orthossteric and the allosteric parts as well as the linker-must be optimized and linked in appropriate ways."(101)

\section{REFERENCE}

101. Mohr K, Schmitz J, Schrage R, Tränkle C, Holzgrabe U: "Molecular Alliance - From Orthosteric and Allosteric Ligands to Dualsteric/Bitopic Agonists at G Protein Coupled Receptors", Angew.Chem. Int. Ed. 2012, 51:2-11.
The online version of the original article can be found at http:// dx.doi.org/10.1208/s12248-015-9776-y.

\footnotetext{
${ }^{1}$ Department of Pharmaceutical Sciences and Computational Chemical Genomics Screening Center, School of Pharmacy, 3501 Terrace Street, 529 Salk Hall, Pittsburgh, Pennsylvania 15261, USA.

${ }^{2}$ NIDA National Center of Excellence for Computational Drug Abuse Research, Pittsburgh, Pennsylvania, USA.

${ }^{3}$ Drug Discovery Institute, Pittsburgh, Pennsylvania, USA.

${ }^{4}$ Departments of Computational Biology and of Structural Biology, University of Pittsburgh, Pittsburgh, Pennsylvania 15261, USA.

${ }^{5}$ To whom correspondence should be addressed. (e-mail: xix15@pitt.edu)
} 